\title{
BLEVE: Theory and Importance in Oil Recovery
}

\author{
Anthony A. Clifford* \\ Department of Chemistry, University of Leeds, Leeds LS2 9JT, UK
}

\begin{abstract}
BLEVEs as phenomena are defined as extremely violent explosions that may occur when liquids and highdensity fluids under pressure are subject to a failure in the containing vessel. Some examples are given and the theory of BLEVEs explained. Oil recovery and carbon capture and storage have made BLEVE relevant today.
\end{abstract}

\section{INTRODUCTION}

BLEVEs are extremely violent explosions that occur very infrequently. They can occur with liquids and high-density fluids under pressure when there is a failure in the containing vessel. The acronym stands for Boiling Liquid Expanding Vapour Explosion, which is probably a misnomer in view of the explanation below. The explosions are orders of magnitude more violent than most explosions. Strong steel containers are highly distorted, flattened, broken into pieces and fragments propelled hundreds of metres. Fire or other forms of heating may be involved, but not necessarily. The premises in which the tanks were located are completely destroyed.

The subject has current relevance because of the use of carbon dioxide, which is prone to BLEVE under commonly used conditions, because of its use in oil recovery and carbon capture and storage (CCS). Consequently, most of the examples of calculation in this paper are of carbon dioxide and its mixtures with methane.

The term BLEVE is most often used to describe explosions in propane tankers following a fire. Particular examples may or may not be true BLEVEs [1]. Explosions involving flammable substances like propane involve a fireball, whereas those involving inflammable substances do not, although they can be just as dramatic. The study of these explosions is difficult because of the element of chance involved in the process. Other examples involve carbon dioxide and water. The most catastrophic example for this gas was the explosion of a tank of carbon dioxide at a plant in Worms, Germany [2]. A tank of 30 tonne capacity was shattered into a number of pieces and only $20 \%$ of the tank was present in the original premises after the explosion. Most of the tank was propelled 300 metres into the Rhine. There were three fatalities and a further eight casualties.

Why do BLEVEs occur? There does not yet appear to be universal agreement on the answer to this question. However, a theory developed by Professor Reid and colleagues at MIT [3] shows that a very dramatic physical event must occur under certain thermodynamic conditions, and this is

*Address correspondence to this author at the Department of Chemistry, University of Leeds, Leeds LS2 9JT, UK; Tel: 01423325 238;

Fax: 01423325 296; E-mails: tony @ criticalprocesses.com,

A.A.Clifford@chem.leeds.ac.uk likely to be the explanation (or at least one of the explanations) of BLEVEs. A brief explanation of this theory can be given with the aid of Fig. (1), which is a diagram of the relationship between the pressure in a substance and the volume it occupies as a liquid, gas or fluid at constant temperature, i.e. the $p-V$ cross-section of the phase diagram. These isotherms were first observed for carbon dioxide by Thomas Andrews, working in the north of Ireland.

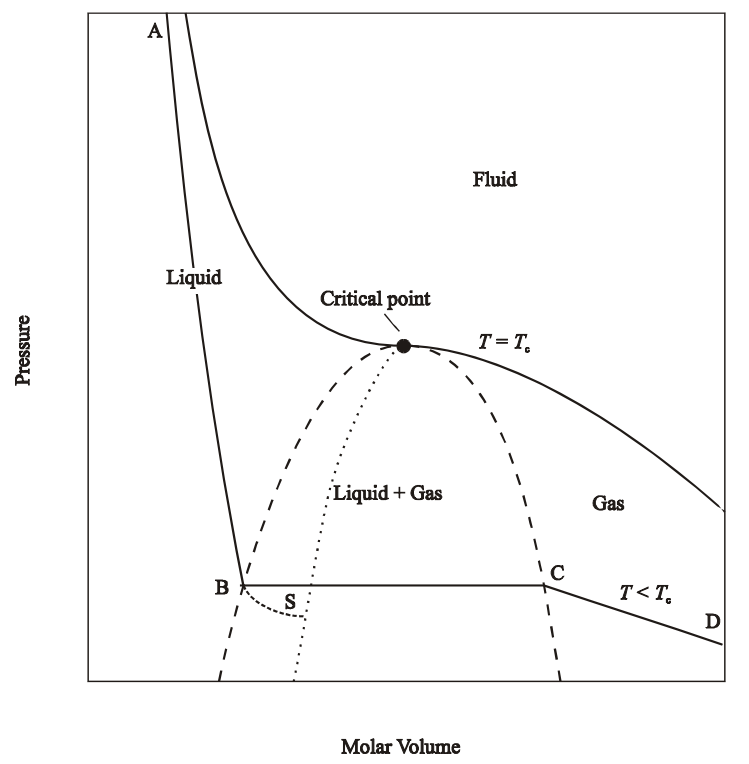

Fig. (1). Variation of pressure with volume occupied at constant temperature and equilibrium shown as solid lines: the higher at the critical temperature and the lower at a temperature below critical. The metastable situation is shown as the dashed line BS and the spinodal curve as the dotted line.

The continuous thick black line ABCD shows the behaviour of the substance at a constant temperature and at thermodynamic equilibrium. In the section $\mathbf{A B}$, the substance is a liquid and as the volume it occupies is expanded the pressure falls dramatically. Eventually the pressure falls to the vapour pressure of the liquid at the particular temperature at B. The liquid then starts to evaporate to become a liquid-gas 
mixture, and the pressure stays constant at the vapour pressure. Eventually it reaches $\mathbf{C}$, where the liquid has been completely converted to gas. The pressure then drops as it is expanded further.

However, if the pressure falls suddenly, perhaps due to a failure in the container, the substance can become an unstable liquid along the dashed path BS. Along the path BS the substance is metastable and can at any time boil to return to the equilibrium horizontal line BC. Although such an event can be very violent it is not thought to be a BLEVE and is described as 'bumping'. Typically violent boiling will occur before the point $\mathbf{S}$ is reached and a BLEVE will not occur, unless further rupture happens. (In practice these events can often be complex.)

One way of making it less probable that the spinodal curve will be reached is to arrange for the drop in pressure following failure to be slower, which can be done in some situations. If the fall in pressure is slower this gives more time for the system to move from the metastable curve BS on to the equilibrium curve BC. This can be achieved by, for example, by attaching a tube to the outlet of a bursting disc to slow the rate of exit of gas.

However, in the unlikely event that the point $\mathbf{S}$ is reached a special and catastrophic situation arises. $\mathbf{S}$ is known as a spinodal point and the slope of the line at this point is zero (i.e. $\left.(\partial p / \partial V)_{T}=0\right)$. The dotted line connects these points at different temperatures and is known as the spinodal curve, which ends at the critical point. The special nature of situations represented by points along this curve are that large density fluctuations can occur because of the insensitivity of pressure to volume $\left((\partial p / \partial V)_{T}=0\right)$. This is because random molecular motion causes molecules to move excessively into some regions and sparsely into others. These densities are normally smoothed out by pressure differences between the high- and low-density regions. However, because $(\partial p / \partial V)_{T}=0$ there is no pressure force to do this. These density fluctuations cannot easily be observed for obvious practical reasons except near the critical point, where the phenomenon is known as critical opalescence.

Once the spinodal curve is reached separation into liquid and gas must occur and rapidly. The density variations develop spontaneously into liquid and gas regions. This occurs homogeneously throughout the whole liquid. The rise in pressure on to the vapour pressure line $\mathbf{B C}$ is not large but it happens at great speed, homogeneously and at the time-scale of molecular motion. The shock to the containing vessel is huge and a disastrous BLEVE happens.

For a BLEVE to occur, the substance has therefore to find itself on the spinodal curve. As practical conditions do not normally allow pressures below atmospheric, the system must hit the spinodal curve between 1 bar or thereabouts and the critical point where the curve ends. When a catastrophic failure occurs there is not time for heat to pass into the system and so the path during failure is adiabatic. We must then assume that a smooth pressure drop occurs the rupture and therefore the pressure drop will be reversible, and thus not only adiabatic but also isentropic. Thus for a BLEVE to occur the entropy of the system before failure must be below the entropy at the critical point but above the entropy on the spinodal curve at 1 bar.

It is therefore possible to calculate the range of temperatures and pressures where the gas or gas mixture would reach this section of the spinodal curve following catastrophic failure. This range is represented by an envelope in temperaturepressure space conveniently drawn on a diagram where the abscissa (x-axis) represents temperature. The right-hand (higher temperature) boundary of this envelope represents temperature-pressure conditions where the entropy equals the critical entropy. To the right of this boundary the entropy will be above the critical entropy and therefore not subject to BLEVE. The critical point will lie on this boundary. The left-hand (lower temperature) boundary of this envelope represents temperature-pressure conditions where the entropy equals the entropy on the spinodal curve at $1 \mathrm{bar}$. To the left of this boundary the entropy will be below the spinodal entropy at 1 bar and therefore not subject to BLEVE.

If there are two phases already present, as in a carbon dioxide gas cylinder or fire extinguisher, BLEVE should not occur as the sudden development of two phases cannot happen. However, there is some evidence from propane experiments that BLEVE can occur in the bulk of the liquid even though there is a vapour phase above it.

\section{CALCULATION OF CONDITIONS FOR BLEVE}

The Stryjek and Vera modification of the Peng-Robinson equation of state (PRSV) was used for all calculations [4]. This gives the pressure, $p$, from the molar volume, $V$, temperature, $T$, gas constant, $R$, and two parameters $a$ and $b$.

$$
\begin{aligned}
& p=\frac{R T}{V-b}-\frac{a}{V^{2}+2 b V-b^{2}} \\
& b=0.077796 R T_{\mathrm{c}} / p_{\mathrm{c}} \\
& a=a\left(T_{\mathrm{c}}\right) \alpha \\
& a\left(T_{\mathrm{c}}\right)=0.457235 R^{2} T_{\mathrm{c}}^{2} / p_{\mathrm{c}} \\
& \alpha=\left[1+\kappa\left(1-\left(T / T_{\mathrm{c}}\right)^{1 / 2}\right)\right]^{2} \\
& \kappa=\kappa_{0}+\kappa_{1}\left(1-\left(T / T_{\mathrm{c}}\right)^{1 / 2}\right)\left(0.7-\left(T / T_{\mathrm{c}}\right)\right) \\
& \kappa_{0}=0.378893+1.48971 \omega-0.17131848 \omega^{2}+0.0196554 \omega^{3}
\end{aligned}
$$

The basic parameters used for the components were also taken from Stryjek and Vera [4] and are shown in Table 1.

For the mixtures the $a_{i}$ and $b_{i}$ values are calculated for each component from the equations for $a$ and $b$ above They are then combined to give values for $a$ and $b$ for the mixture using the following combining rules, where $x_{i}$ are the mole fractions of the components and $k_{i j}$ the binary interaction parameter.

$$
\begin{aligned}
a & =\sum_{i} \sum_{j} x_{i} x_{j}\left(a_{i} a_{j}\right)^{1 / 2}\left(1-k_{i j}\right) \\
b & =\sum_{i} x_{i} b_{i}
\end{aligned}
$$

The binary interaction parameter for methane and carbon dioxide was taken to be 0.1 . 
The entropy, $S_{0}(298.14)$, of the ideal gas at $298.14 \mathrm{~K}$ and 1 atmosphere (1.01325 bar) was taken to be zero. It was not necessary to take into account the entropy of mixing as the calculation involved comparison of entropies at different conditions of the same mixture. To obtain absolute entropies the absolute entropy at $298.14 \mathrm{~K}$ and 1 bar must be added to the results here. E.g. for carbon dioxide $213.7 \mathrm{~J} \mathrm{~K}^{-1} \mathrm{~mol}^{-1}$ must be added. The entropy was for temperature $T$. is then equal to the quantity $\int_{298.14}^{T} \frac{C_{p}}{T} \mathrm{~d} T$, where $C_{p}$ is the heat capacity at constant pressure. This was obtained from the equation $C_{p} / R=a_{0}+a_{1} T+a_{2} T^{2}+a_{3} T^{3}+a_{4} T^{4}$, using published values [6] given in Table 2 for the parameters $a_{0}$ $a_{4}$. The entropy was then corrected for pressure $p$ (in bar), still as an ideal gas, by adding the quantity $-R \ln p$. It is then corrected for non-ideality subtracting the departure function obtained from the PRSV equation of state, which is given below.
$-R \ln \frac{p V}{R T}(1-b / V)+\frac{\mathrm{d} a / \mathrm{d} T}{\sqrt{8} b} \ln \frac{V+b(1-\sqrt{2})}{V+b(1+\sqrt{2}}$

After these terms are combined, the entropy, $S$, could be obtained (relative to the entropy of the ideal mixture at 298.14 K and 1 bar).

For the mixtures, the critical points were obtained. This was done by calculating $p$ and $(\partial p / \partial V)_{T}$ for a range of values of $V$, making sure finally that this range included $V_{\mathrm{c}}$. The temperature was then adjusted to find the lowest value where $(\partial p / \partial V)_{T}$ was always negative, i.e. was just inside the supercritical state. This value is shown in Table $\mathbf{3}$ as the critical temperature, $T_{\mathrm{c}}$. The critical point was then taken as the lowest value of $(\partial p / \partial V)_{T}$ (in theory zero) and $V$ and $p$ at this point were taken as $V_{\mathrm{c}}$ and $p_{\mathrm{c}}$ and shown in Table $\mathbf{3}$. Table 3 also contains for completeness the critical parameters of the pure gases. For the pure gases and mixtures, the entropy was then calculated at the critical point. These data are shown in Table 3 .

Table 1. Basic Parameters for the Components. $T_{\mathrm{c}}, p_{\mathrm{c}}$ and $V_{\mathrm{c}}$ are the Critical Temperature, Pressure and Molar Volume, Respectively. $\omega$ is the Acentric Factor and $\kappa_{1}$ the Additional Stryjek and Vera Parameter [4]

\begin{tabular}{|c|c|c|c|c|c|}
\hline & $\boldsymbol{T}_{\mathbf{c}}(\mathbf{K})$ & $\boldsymbol{p}_{\mathbf{c}}(\mathbf{b a r})$ & $\boldsymbol{V}_{\mathbf{c}} \times \mathbf{1 0}^{\mathbf{5}}\left(\mathbf{m}^{\mathbf{3}} \mathbf{m o l}^{\mathbf{1}}\right)$ & $\boldsymbol{\omega}$ & $\mathbf{K}_{\mathbf{1}}$ \\
\hline \hline Methane & 190.555 & 45.9500 & 9.860 & 0.0105 & $-0.00159^{*}$ \\
\hline Propane & 369.820 & 42.4953 & 20.000 & 0.1542 & $0.03136^{*}$ \\
\hline $\mathrm{CO}_{2}$ & 304.210 & 73.8243 & 9.407 & 0.2250 & $0.04285^{*}$ \\
\hline
\end{tabular}

$*=0$ above $0.7 T_{\mathrm{c}}$.

Table 2. Parameters Used for Heat Capacity Calculations [5]

\begin{tabular}{|c|c|c|c|c|c|}
\hline & $\boldsymbol{a}_{0}$ & $\boldsymbol{a}_{\mathbf{1}}$ & $\boldsymbol{a}_{\mathbf{2}}$ & $\boldsymbol{a}_{3}$ & $\boldsymbol{a}_{\mathbf{4}}$ \\
\hline \hline Methane & 4.568 & $-8.975 \mathrm{E}-03$ & $3.631 \mathrm{E}-05$ & $-3.407 \mathrm{E}-08$ & $1.091 \mathrm{E}-11$ \\
\hline Propane & 3.847 & $5.131 \mathrm{E}-03$ & $6.011 \mathrm{E}-05$ & $-7.893 \mathrm{E}-08$ & $3.079 \mathrm{E}-11$ \\
\hline $\mathrm{CO}_{2}$ & 3.259 & $1.356 \mathrm{E}-03$ & $1.502 \mathrm{E}-05$ & $-2.374 \mathrm{E}-08$ & $1.056 \mathrm{E}-11$ \\
\hline
\end{tabular}

Table 3. Thermodynamic Values for the Critical Point and Spinodal Point at 1 Bar

\begin{tabular}{|c|c|c|c|c|c|c|}
\hline & & $\mathrm{CO}_{2}$ & Propane & Methane & $75 \% \mathrm{CO}_{2} 25 \% \mathrm{CH}_{4}$ & $50 \% \mathrm{CO}_{2} 50 \% \mathrm{CH}_{4}$ \\
\hline \multirow[t]{4}{*}{ Critical point } & $p_{\mathrm{c}}(\mathrm{bar})$ & 73.95 & 42.50 & 45.95 & 66.01 & 58.51 \\
\hline & $V_{\mathrm{c}} \times 10^{5}\left(\mathrm{~m}^{3} \mathrm{~mol}^{-1}\right)$ & 9.41 & 20.00 & 9.86 & 10.55 & 10.57 \\
\hline & $T_{\mathrm{c}}(\mathrm{K})$ & 304.21 & 369.82 & 190.56 & 272.30 & 241.82 \\
\hline & $S_{\mathrm{c}}\left(\mathrm{J} \mathrm{K}^{-1} \mathrm{~mol}^{-1}\right)$ & -56.31 & -32.42 & -64.22 & -56.5 & -58.76 \\
\hline \multirow[t]{4}{*}{ Spinodal point at $1 \mathrm{bar}$} & $p$ (bar) & 1.00 & 1.00 & 1.00 & 1.00 & 1.00 \\
\hline & $V \times 10^{5}\left(\mathrm{~m}^{3} \mathrm{~mol}^{-1}\right)$ & 6.45 & 13.60 & 6.50 & 6.46 & 6.47 \\
\hline & $T(\mathrm{~K})$ & 278.50 & 337 & 171.3 & 248.15 & 219.35 \\
\hline & $S\left(\mathrm{~J} \mathrm{~K}^{-1} \mathrm{~mol}^{-1}\right)$ & -67.25 & -49.0 & -74.3 & -68.9 & -70.66 \\
\hline
\end{tabular}


Next, for all gases and mixtures, the point on the spinodal curve at 1 bar was obtained by again calculating $p$ and $(\partial p / \partial V)_{T}$ for a range of values of $V$ and adjusting the temperature to find a value of $V$ where $p$ was 1 bar and $(\partial p / \partial V)_{T}$ was close to zero and changing from negative to positive (i.e. $p$ was going through a minimum, indicating a spinodal point). The values of $p, V$ and $T$ for these spinodal points are given in Table 3. The entropies were then calculated and also shown in Table $\mathbf{3}$.

The right-hand boundary of the BLEVE envelopes was then calculated. This was done for a range of temperatures by adjusting $V$ so that the entropy was equal to the critical entropy, and then calculating the pressure. The temperature range was chosen to obtain pressures between 400 bar and the bottom angle of the BLEVE envelope. Calculations were carried out at $10^{\circ} \mathrm{C}$ intervals. The left-hand boundary was then calculated similarly, adjusting to the entropy at the spinodal point at 1 bar. The results are shown in Table 4.

\section{DISCUSSION OF THE RESULTS}

Fig. (2) shows the calculated BLEVE envelope for carbon dioxide as the thick black lines. It is compared with that calculated by Kim and Reid [3], which has been scanned into the figure. The curves are similar but do not exactly coincide. This is because Kim and Reid used a different method for obtaining entropies. They used the entropy values of reference [6] (a numerical correlation of experimental values) and adjusted them to the conditions required using departure functions. An analytical equation of state is used here, which is less accurate but more universally applicable, especially for mixtures. For carbon dioxide the BLEVE envelope is in

Table 4. Numerical Results for the BLEVE Envelope Pressure-Temperature Limits for the Pure Gases and Mixtures. LH and RH are the Left-Hand and Right Hand Boundaries the Envelope, Respectively. The RH Limit is Calculated from the Entropy at the Critical Point and the LH Limit is Calculated from the Entropy on the Spinodal Curve at 1 Bar

\begin{tabular}{|c|c|c|c|c|c|c|c|c|c|c|}
\hline \multirow[b]{2}{*}{ Temp. $\left({ }^{\circ} \mathbf{C}\right)$} & \multicolumn{10}{|c|}{ Pressure (Bar) } \\
\hline & \multicolumn{2}{|c|}{$\mathrm{CO}_{2}$} & \multicolumn{2}{|c|}{ Propane } & \multicolumn{2}{|c|}{ Methane } & \multicolumn{2}{|c|}{$75 \% \mathrm{CO}_{2} 25 \% \mathrm{CH}_{4}$} & \multicolumn{2}{|c|}{$50 \% \mathrm{CO}_{2} 50 \% \mathrm{CH}_{4}$} \\
\hline-90.0 & & & & & 35.78 & 45.30 & & & & \\
\hline-60.0 & & & & & 93.23 & 259.23 & & & & \\
\hline-50.0 & & & & & 123.97 & 372.83 & & & & \\
\hline-40.0 & & & & & 162.23 & 513.11 & & & 47.18 & 67.56 \\
\hline-30.0 & & & & & 209.20 & & & & 60.45 & 133.31 \\
\hline-20.0 & & & & & 266.22 & & & & 77.26 & 217.04 \\
\hline 20.0 & 54.54 & 64.57 & & & & & 106.11 & 276.42 & 194.97 & \\
\hline 30.0 & 71.87 & 121.44 & & & & & 131.63 & 380.24 & 241.57 & \\
\hline 40.0 & 92.98 & 191.14 & & & & & 162.08 & 504.75 & 297.21 & \\
\hline 50.0 & 118.42 & 275.59 & & & & & 198.17 & & 363.21 & \\
\hline 60.0 & 148.83 & 376.91 & & & & & 240.70 & & 441.02 & \\
\hline 70.0 & 184.92 & & & & & & 290.55 & & 60.45 & 133.31 \\
\hline 80.0 & 227.46 & & 33.24 & 44.52 & & & 348.72 & & 77.26 & 217.04 \\
\hline 90.0 & 277.33 & & 38.07 & 95.65 & & & 416.31 & & & \\
\hline 100.0 & 335.49 & & 45.19 & 174.67 & & & & & & \\
\hline 170.0 & & & 376.54 & & & & & & & \\
\hline 180.0 & & & 531.57 & & & & & & & \\
\hline
\end{tabular}


the region where conditions are typically used in processes. Since the disaster referred to earlier, carbon dioxide is always stored cooled at atmospheric pressure or where two phases are present. However for carbon capture and storage processing of carbon dioxide under pressure will be required and it will be prudent to avoid the BLEVE envelope. Smallscale experiments have been carried out to attempt to produce BLEVE in circumstances when BLEVE could occur $[3,7]$, but it could not be made to happen. It was believed that boiling occurred before the spinodal curve was reached.

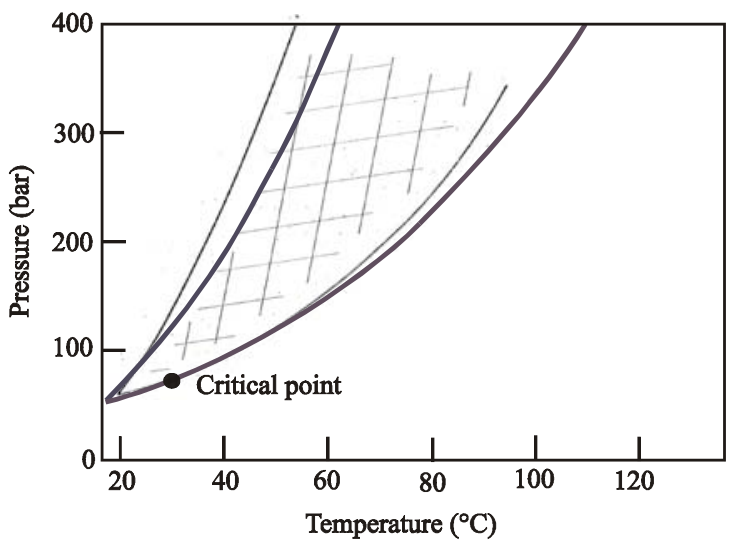

Fig. (2). The BLEVE envelope for pure carbon dioxide, shown as the solid black lines. The results of Kim and Reid [3] are shown for comparison.
Fig. (3) compares the BLEVE envelopes for methane, carbon dioxide and propane. As the critical point is on the BLEVE envelope, the curves follow the critical temperatures. Thus for propane higher temperatures are reached and that is believed to be the reason that propane BLEVEs occur in fires. For methane the BLEVE envelope is at lower temperatures, removed from normal ambient conditions. However when natural gas is transported as a liquid, it is cooled and it is important to avoid the BLEVE envelope. Fig. (4) shows the BLEVE envelopes for mixtures of carbon dioxide and methane, which lie between those of the pure gases. Curves like these must be considered when designing processes for the recovery of natural gas using carbon dioxide. Also, as the BLEVE envelopes are predicted to be wider for mixtures [8], the curves overlap.

To summarise, we have a situation where very catastrophic explosions occasionally occur, which are orders of magnitude more violent than most explosions. At the same time we have a theory based on physical principles, which predicts that in some circumstances a phenomenon may occur to produce these huge explosions. However, what we don't have is an experimental study showing that the theory and the physical manifestations are definitely related. Experiments on carbon dioxide did not result in BLEVEs and the study for propane did not seem to accord with the theory. Nevertheless, it is very likely that the Reid theory does explain at least some BLEVEs, although other theories may be valid in some cases.

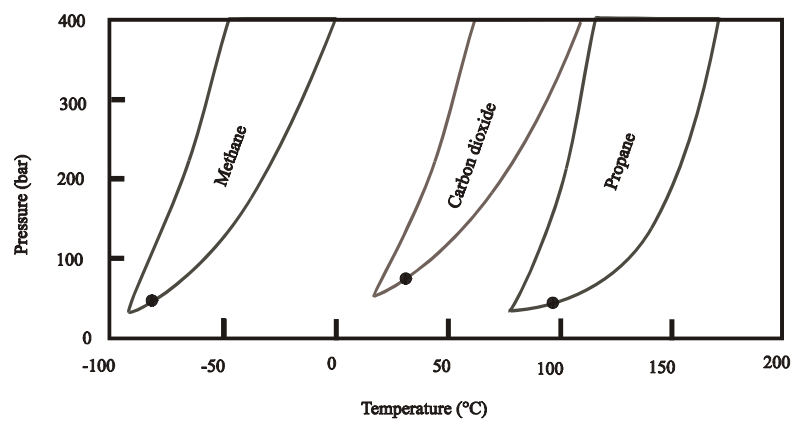

Fig. (3). The BLEVE envelopes for methane, carbon dioxide and propane.

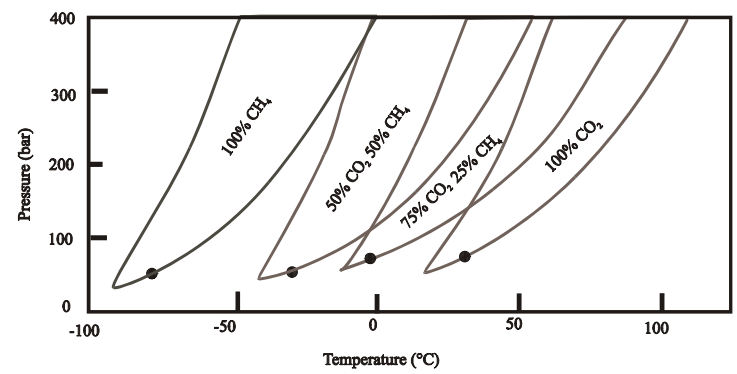

Fig. (4). The BLEVE envelopes for methane, carbon dioxide and two mixtures. 
In conclusion for companies operating processes involving gases under pressure, BLEVE calculations should be carried out and the BLEVE envelope avoided during operations. If this is not done, the chances of a BLEVE event are still small but, if it does happen, the consequences would be catastrophic.

\section{REFERENCES}

[1] http://me.queensu.ca/people/birk/research/thermalHazards/bleve/, 22/07/2008.

[2] W. E. Clayton and M. L. Griffin, "Catastrophic failure of a liquid carbon dioxide storage vessel", Process Saf. Prog., vol. 13, pp. 202-209, October 1994

[3] M. E. Kim and R. C. Reid, "The rapid depressurization of hot, high pressure liquids or supercritical fluids", in Chemical Engineering at
Supercritical Fluid Conditions, M. E. Paulaitis, Ed. Ann Arbor: Ann Arbor Science, 1983, pp. 81-100.

[4] R. Stryjek and J. H. Vera, "PRSV - an improved Peng-Robinson equation of state for pure compounds and mixtures", Can. J. Chem. Eng., vol. 64, pp. 323-333, April 1986.

[5] B. E. Poling, J. M. Prausnitz, and J. P. O'Connell, The Properties of Gases and Liquids, $5^{\text {th }}$ ed, New York: McGraw-Hill, 2000.

[6] S Angus, B. Armstrong and K. M. de Reuch, International Thermodynamic Tables of the Fluid State - Vol. 3 Carbon Dioxide. Oxford: Pergamon Press, 1976.

[7] H. Z. Li and M. Perrut, "Flash discharge of supercritical fluid and high pressure gas through pipes: experimental results and modelling", Chem. Eng. Commun., vol. 117, pp. 415-431, September 1992.

[8] B. L. Beegle, M. Modell and R. C. Reid, "Thermodynamic stability criteria for pure substances and mixtures", AIChE J., vol.20, pp. 1200-1206, November 1974.

() Anthony A. Clifford; Licensee Bentham Open.

This is an open access article licensed under the terms of the Creative Commons Attribution Non-Commercial License (http://creativecommons.org/licenses/by$\mathrm{nc} / 3.0 /$ ) which permits unrestricted, non-commercial use, distribution and reproduction in any medium, provided the work is properly cited. 